\title{
Semi-Circular Potential Sweep Voltammetry: Electrochemically Quasi-Reversible System
}

\author{
Kyungsoon Park and Seongpil Hwang* \\ Department of Advanced Materials Chemistry, Korea University, Sejong 30019, Korea
}

\begin{abstract}
The novel voltammetry using a semi-circular potential wave for quasi-reversible charge transfer system on electrode is theoretically investigated. Compared with conventional voltammetry based on linear sweep such as linear sweep voltammetry (LSV), semi-circular potential sweep voltammetry (SCV) may decrease the charging current outside the center of potential range and increase the faradaic current at the midpoint due to variable scan rate. In this paper, we investigate the system based on macroelectrode where simple 1 dimensional $(1 \mathrm{D})$ diffusion system is valid with various charge transfer rate constant $\left(k_{0}\right)$. In order to observe the amplification at midpoint, voltammetric response with different midpoint ranging from $-200 \mathrm{mV}$ to $200 \mathrm{mV}$ are studied. SCVs shows both the shift of peak potential and the amplification of peak current for quasi-reversible electrode reaction while only higher peak current is observed for reversible reaction. Moreover, the higher current at midpoint enable the amplification of current at low overpotential region which may assist the determination of onset potential as a figure-of-merit in electrocatalyst.
\end{abstract}

Keywords : Linear Sweep Voltammetry, Cyclic Voltammetry, Semi-Circular Wave Form, Quasi-Reversible Charge Transfer, Onset Potential

Received : 22 April 2020, Accepted : 29 June 2020

\section{Introduction}

Voltammetry, especially cyclic voltammetry (CV) and linear sweep voltammetry (LSV), is one of basic and fundamental methods in order to characterize various electrochemical system ranging from redox couples to energy conversion/storage. They can provide information on thermodynamics, kinetics, reaction mechanism, and mass transportation. Conventionally, CV and LSV use a linear potential sweeps, which enable the estimation of charge transfer rate, concentration of redox molecules, diffusion coefficient, formal potential, onset potential and so on. A distinguishing feature of typical CV and LSV is a constant scan rate originated from a linear sweep over entire electrochemical measurement. Thus, the current response of voltammetry can be modeled by the sum of faradic current dependent on square root of scan rate and of

*E-mail address: sphwang@korea.ac.kr

DOI: https://doi.org/10.33961/jecst.2020.00962

This is an open-access article distributed under the terms of the Creative Commons Attribution Non-Commercial License (http://creativecommons.org/licenses/by-nc/4.0) which permits unrestricted non-commercial use, distribution, and reproduction in any medium, provided the original work is properly cited. charging current proportional to scan rate. Although well-developed theory on a linear potential sweeps makes these voltammetry popular in science, the interpretation of voltammetric data requires extensive training. Unfortunately many papers were unintentionally misleaded the electrochemical results due to the lack of deep insight on voltammetry. One of example is onset potential which have been widely used as a figure of merit in order to estimate electrocatalyst or photoelectrode. Onset potential or voltage is considered as the voltage where current start to increase from background current in engineering. Classical Butler-Volmer model describes the charge transfer by the summation of forward and reverse reaction whose form is the exponential function written in the following.

$$
i=i_{o}\left[e^{-\alpha f n}-e^{(1-\alpha) f \eta}\right]
$$

Where is the exchange current, is the transfer coefficient, $f$ is the and . Thus, onset potential cannot be defined precisely from this model owing to exponential expression. Nonetheless, we agree that onset 
potential is a simple and intuitive estimator to compare the charge transfer kinetics for a laymen in electrochemistry which become a de fact standard in society. The determination of onset potential from voltammetry, however, is still unclear.

Recently Compton group suggested the voltammetry with non-triangular or non-linear potential sweep such as cosine [1], cosine square [2], and semi-circular potential waveform [3]. We are interested in semicircular potential sweep because it provides variable scan rate over potential range in contrast to the feature of constant scan rate in typical voltammetry. That is an instantaneous infinite scan rate at the midpoint and a much smaller scan rate except the midpoint of potential range for electrochemistry. This unique feature amplifies both faradic current and nonfaradic current at midpoint and attenuate them outside of midpoint. Compton group proposed that the semi-circular potential sweep voltammetry can be applied to the precise determination of formal potential from LSV [4] and the determination of low concentration analyte [5].

Herein we report the theoretical investigation of semi-circular potential sweep voltammetry for quasireversible charge transfer system on electrode. Previous reports on semi-circular potential sweep voltammetry studied the theory on electrode for reversible charge transfer $[3,4]$, the simulation on microelectrode for quasi-reversible system [6], and the experimental verification [7]. Our system is based on macroelectrode where electrode area is sufficiently large neglecting edge effect in diffusion layer thickness. Thus simple 1 dimensional (1 D) diffusion system is adopted with various charge transfer rate constant $\left(k_{0}\right)$. Voltammetric behaviors of semi-circu-

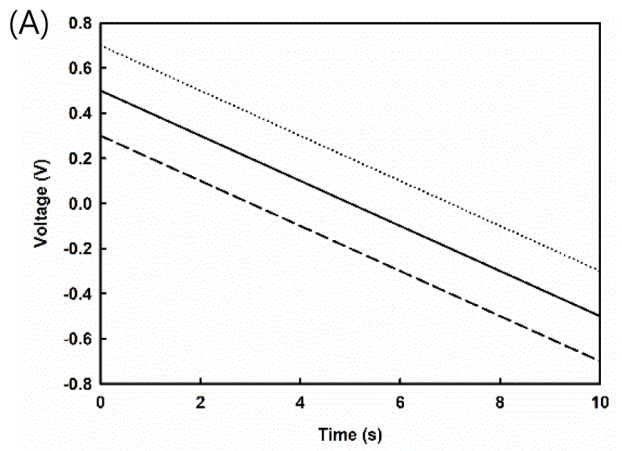

lar potential sweep are compared with that of linear sweep to show the unique features of amplification at midpoint. Moreover, the effect of midpoint for relatively sluggish charge transfer is investigated to find the better electrochemical method to verify onset potential.

\section{Experimental}

All results were obtained using COMSOL Multiphysics 5.4 with Electrochemistry Module to simulate the voltammetric response. The diffusion equation in simple 1D system was solved with Butler-Volmer model as a charge transfer reaction. 2 species of $\mathrm{Ox}$ and Red was considered whose standard reduction potential was assumed 0 . One-electron transfer redox couple Ox/Red with different $k_{0}$ were investigated. Identical diffusion coefficient of $\mathrm{Ox}$ and Red were assumed as $D_{\mathrm{Ox}}=\mathrm{D}_{\mathrm{Red}}=\mathrm{D}=1 \times 10^{-10} \mathrm{~m}^{2} / \mathrm{s}$ at $\mathrm{T}=298 \mathrm{~K}$. Double layer capacitance was assumed as $0.2 \mathrm{~F} / \mathrm{m}^{2}$. Initial concentration of $\mathrm{Ox}$ was set at $1 \mathrm{mM}$ while that of Red was at $0 \mathrm{mM}$. For accuracy, time step in solver of COMSOL was set to constant value manually.

\section{Results and Discussion}

As a reminder, LSV applied the linear potential waveform to working electrode written in the following.

$$
\mathrm{V}(t)=\mathrm{V}(t=0) \pm v t+\mathrm{V}_{\text {cen }}
$$

Where $\mathrm{V}(t=0)$ is the initial potential and $v$ is the scan rate. Solid line in Fig. 1(A) shows the linear sweep potential from $0.5 \mathrm{~V}$ to $-0.5 \mathrm{~V}$ with the scan

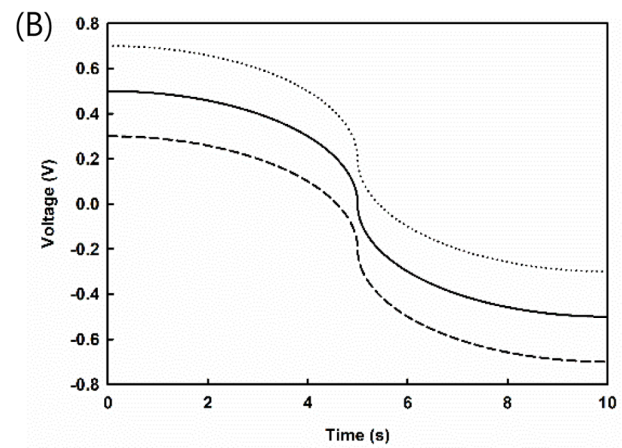

Fig. 1. Time $v s$. the applied potential of (A) Linear sweep voltammetry (LSV) and (B) Semi-circular voltammetry (SCV) with an amplitude of $0.50 \mathrm{~V}$. $\mathrm{V}_{\text {cen }}$ for dotted, solid, and dashed lines are $0.2 \mathrm{~V}, 0 \mathrm{~V}$, and $-0.2 \mathrm{~V}$, respectively. 
rate of $100 \mathrm{mV} / \mathrm{s}$ and $\mathrm{V}_{\text {cen }}=0$ which is conventional scan rate in many electrochemical experiments. Instead of this linear sweep, the semicircular potential sweep is expressed as following.

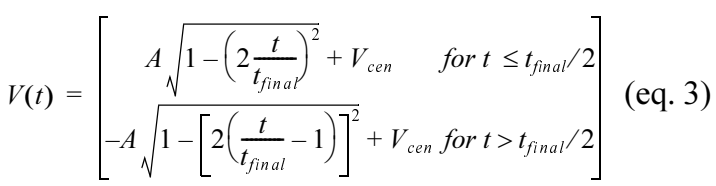

Where $\mathrm{A}$ is the amplitude of potential, $t_{\text {final }}$ is the period of a single scan, and $V_{c e n}$ is the midpoint potential between initial potential and final potential. Fig. 1(B) shows the semicircular potential sweep with the average scan rate of $100 \mathrm{mV} / \mathrm{s}$. It should be mentioned that we express the average scan rate of $100 \mathrm{mV} / \mathrm{s}$ based on the same period of $10 \mathrm{~s}$ even though instantaneous scan rate varies at any point. In addition, $\mathrm{V}_{\text {cen }}$ was swept from $0.2 \mathrm{~V}$ to $-0.2 \mathrm{~V}$ in order to investigate the effect of singular point where scan rate is infinite in principle.

To prove the validity of COMSOL simulation, SCV of reversible electrode reaction was investigated first [4]. Fig. 2 shows (A) conventional LSV and (B) $\mathrm{SCV}$ with different $\mathrm{V}_{\text {cen }}$ with $k_{0}=10^{-3} \mathrm{~m} / \mathrm{s}$. In conventional LSVs, $\mathrm{V}_{\text {cen }}$ do not change any variance in the voltammetric behavior such as onset potential, peak current, peak potential and exponential increment before peak current. In contrast, SCV in Fig. 2(B) demonstrates the huge amplification of current when $\mathrm{V}_{\text {cen }}$ located near 0 . At $\mathrm{V}_{\text {cen }}=-50 \mathrm{mV}$, peak currents increase from ca. $2.7 \mathrm{~mA}$ in LSV to $21.4 \mathrm{~mA}$ in SCV indicating the amplification factor of 7.9. The origin of the amplification is the infinite scan rate at singular point of semi-circular potential at $\mathrm{V}_{\text {cen }}$. Theoretically scan rate is infinite but our results shows finite current caused by limited time step in simulation. This limitation was also observed in previous experimental implementation of SCV [7]. These results are in perfect accordance with previous theoretical and experimental reports by Compton group [4,7], indicating the validity of our approach.

The quasi-reversible electrode reaction by SCV were explored with the same approach. Fig. 3 shows (A) LSVs and (B) SCVs with different $\mathrm{V}_{\text {cen }}$ with $k_{0}$ $=10^{-5} \mathrm{~m} / \mathrm{s}$. LSVs shows slight cathodic shift of peak potential by ca. $90 \mathrm{mV}$ with smaller peak current (from $2.7 \mathrm{~mA}$ to $2.2 \mathrm{~mA}$ ) compared with Fig. 2(A) due to the sluggish charge transfer reaction on electrode. Onset potential, however, seems to be $0.2 \mathrm{~V}$
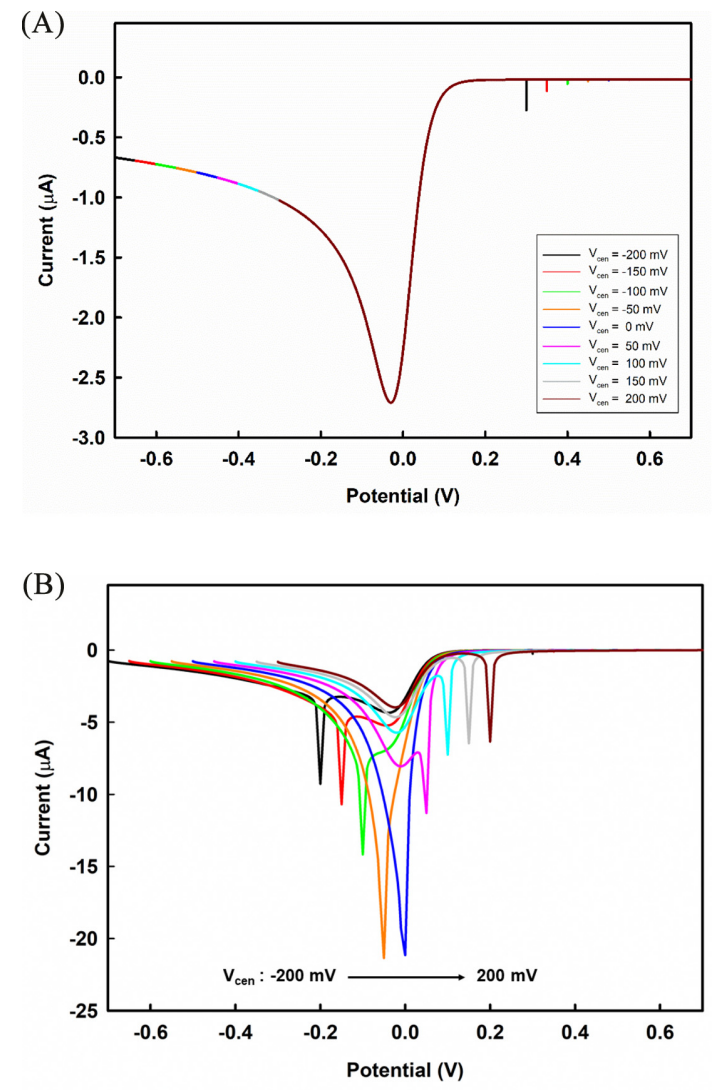

Fig. 2. Voltammetric response from (A) linear sweep potential (LSV) and (B) semi-circular sweep (SCV) using potential waves shown in Fig. 1 with $k_{0}=10^{-3} \mathrm{~m} / \mathrm{s}$. $\mathrm{V}_{\text {cen }}$ varies from $200 \mathrm{mV}$ to $-200 \mathrm{mV}$ with the interval of 50 $\mathrm{mV}$. Inset in (A) are $\mathrm{V}_{\text {cen }}$ value according to the color which applies to all data afterwards.

similar to Fig. 1(A) even much slower charge transfer kinetics. SCVs in Fig. 3(B) shows similar behavior of amplification of current at singular point at $\mathrm{V}_{\text {cen }}$. Maximum amplification of peak current in SCV was ca. 6.3 times at $\mathrm{V}_{\text {cen }}=-150 \mathrm{mV}$, which is smaller than that of reversible charge transfer. This is reasonable because current behaviors over potential sweep are proportional to the square root of scan rate in all of reversible, quasi-reversible and totally irreversible with different coefficients [8]. It is well known that that ratio of peak currents between irreversible and reversible system is the function called $K$ whose value is lower than unit value $[8,9]$. At singular point of SCV, high scan rate enhances the current signal itself but factor is smaller due to the constant function 

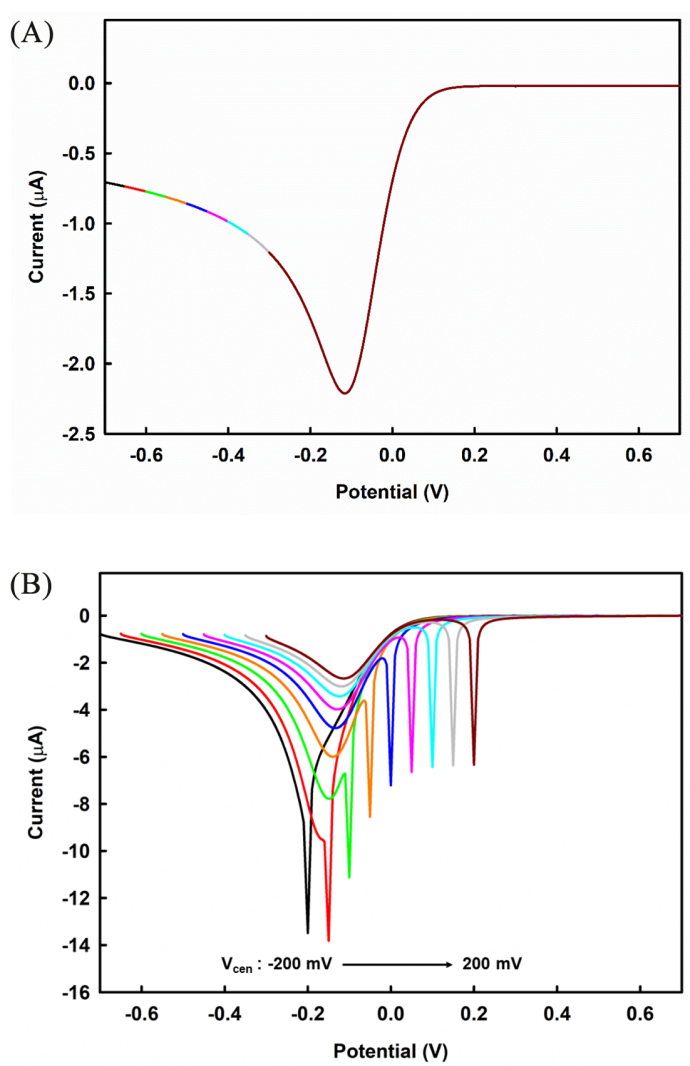

Fig. 3. Voltammetric response from (A) LSV and (B) (SCV) using potential waves shown in Fig. 1 with $k_{0}=10^{-5}$ $\mathrm{m} / \mathrm{s}$. $\mathrm{V}_{\text {cen }}$ varies from $200 \mathrm{mV}$ to $-200 \mathrm{mV}$ with the interval of $50 \mathrm{mV}$.

$K$. The other characteristic results are onset potential, which is an significant figure-of-merit to evaluate electrocatalyst. Shift of onset potential in Fig. 3(A) of LSV for quasi-reversible electrode system is not obvious compared with Fig. 2(A) for reversible system even though $k_{0}$ values differ significantly. In contrast, $\mathrm{SCV}$ with the highest scan rate at $\mathrm{V}_{\text {cen }}$ increase the faradic current resulting in the identification of this difference in the rate constant by current amplification. Onset potential is the starting potential where reduction begins so that current at singular point after onset potential generate much larger current by amplification of faradaic current. We assumed that $5 \%$ change of current at singular point compared with that for non-faradaic current is the indication of onset potential in this report. In order to find the onset potential, the current value at much positive $\mathrm{V}_{\text {cen }}$ $(+200 \mathrm{mV})$ are compared with that at each $\mathrm{V}_{\text {cen }}$ until
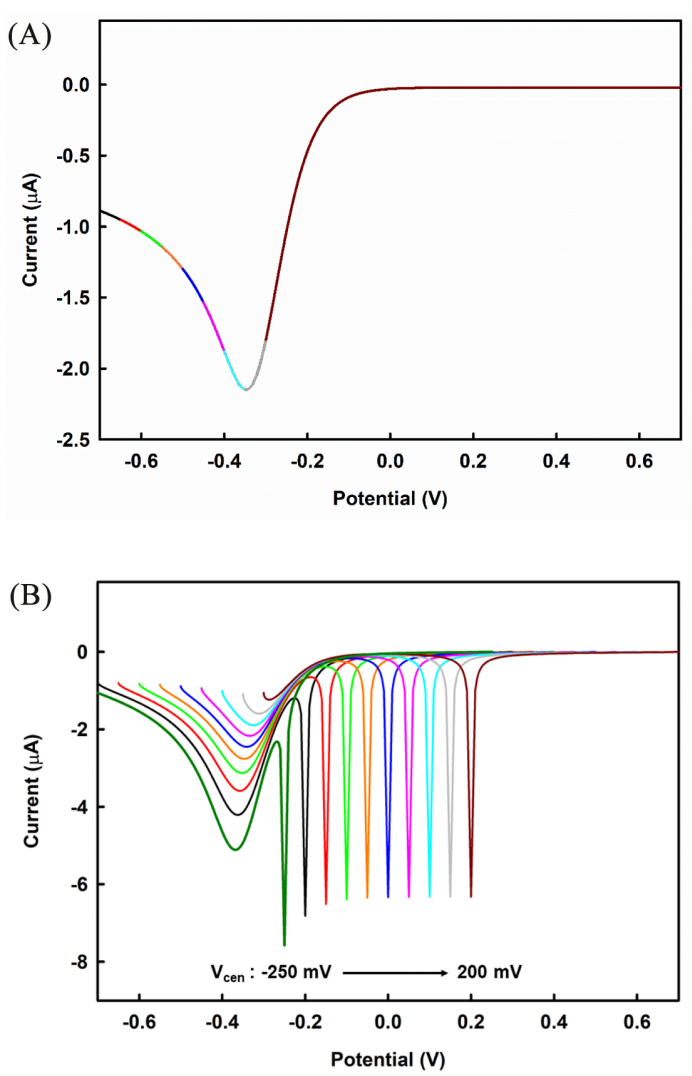

Fig. 4. Voltammetric response from (A) LSV and (B) (SCV) using potential waves shown in Fig. 1 with $k_{0}=10^{-7}$ $\mathrm{m} / \mathrm{s}$. $\mathrm{V}_{\text {cen }}$ varies from $200 \mathrm{mV}$ to $-250 \mathrm{mV}$ with the interval of $50 \mathrm{mV}$. Colors of plots are the same to Figure 2(A) except green for $\mathrm{V}_{\text {cen }}=-250 \mathrm{mV}$.

$5 \%$ change is observed. From the point of this criteria, increase of current at $V_{\text {cen }}$ in Fig. 2(B) is observed near $100 \mathrm{mV}$ as a onset potential while it is around $50 \mathrm{mV}$ in Fig. 3(B). Therefore, current amplification at singular point of SCV offers the more clear indication for onset potential. To make this point more clear, LSVs and SCVs at much smaller charge transfer of $k_{0}=10^{-7} \mathrm{~m} / \mathrm{s}$ were shown in Fig. 4. Singular point of $\mathrm{V}_{\text {cen }}$ is much higher potential than peak potential of LSV so that amplification is just ca. 1.94 (from $2.14 \mathrm{~mA}$ in Figure 4(A) to $4.16 \mathrm{~mA}$ in Fig. 4(B)). Peak potential was also slightly cathodically shifted. Both of peak current and peak potential were originated by faster scan rate of SCV than that of LSV. The onset potential is around $-150 \mathrm{mV}$, indicating the shift of onset potential dependent on charge transfer kinetics. 


\section{Conclusions}

We investigated the voltammetric response a semicircular potential wave for quasi-reversible charge transfer system on electrode. Compared with conventional voltammetry based on linear sweep such as linear sweep voltammetry (LSV), semi-circular potential sweep voltammetry (SCV) may decrease the charging current outside the center of potential range and increase the faradaic current at the midpoint due to variable scan rate. The theoretical simulation on macroelectrode under 1 dimensional diffusion system for reversible charge transfer verified the validity of our simulation from the good accordance with previous reports. For smaller charge transfer rate constant $\left(k_{0}\right)$ with different midpoint ranging from $-200 \mathrm{mV}$ to $200 \mathrm{mV}, \mathrm{SCVs}$ shows both the shift of peak potential and the amplification of peak current for quasi-reversible electrode reaction while only higher peak current is observed for reversible reaction. Moreover, the higher current at midpoint enable the amplification of current at low overpotential region which may assist the determination of onset potential as a figure-of-merit in electrocatalyst.

\section{Acknowledgement}

This work was supported by a Korea University Grant.

\section{References}

[1] E. Katelhon, R.G. Compton, Phys. Chem. Chem. Phys., 2017, 19(42), 28820-28823.

[2] H.M.A. Amin, Y. Uchida, C. Batchelor-McAuley, E. Kätelhön, R.G. Compton, J. Electroanal. Chem., 2018, 815, 24-29.

[3] Y. Uchida, E. Kätelhön, R.G. Compton, J. Electroanal. Chem., 2018, 823, 465-473.

[4] Y. Uchida, E. Kätelhön, R.G. Compton, J. Electroanal. Chem., 2018, 818, 140-148.

[5] Y. Wang, L. Chen, K. Chaisiwamongkhol, R.G. Compton, Food Chem., 2020, 309, 125606.

[6] Y. Uchida, E. Kätelhön, R.G. Compton, J. Electroanal. Chem., 2019, 835, 60-66.

[7] H.M.A. Amin, Y. Uchida, E. Kätelhön, R.G. Compton, J. Electroanal. Chem., 2019, 836, 62-67.

[8] L. R. Faulkner, A. J. Bard, Electrochemical Methods: Fundamentals and Applications, Wiley, New York, 2001, 2(482), 580-632.

[9] H. Matsuda, Y. Ayabe, Z. Elektrochem., 1955, 59(6), 494-503. 